\title{
EOP's: Adherencia al tratamiento en pacientes depresivos luego de un infarto agudo de miocardio
}

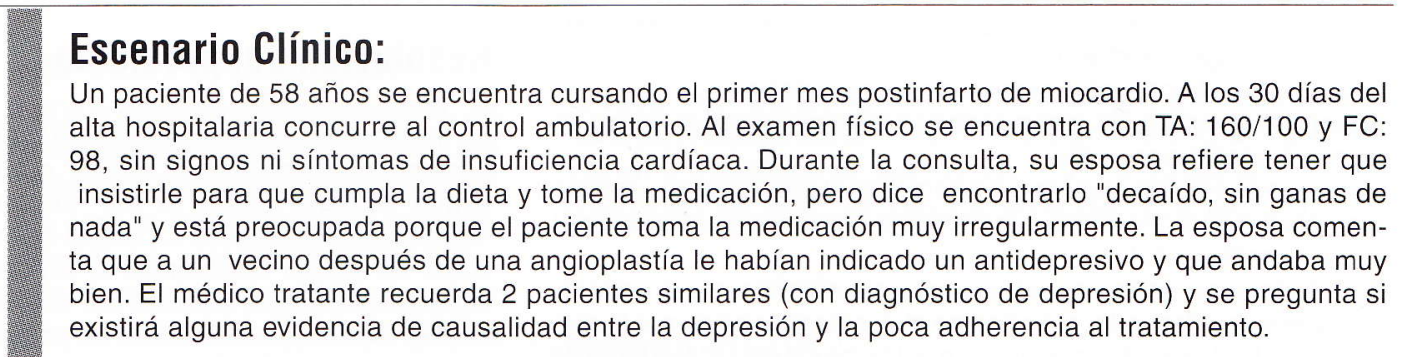

\section{Pregunta que generó el caso}

En los pacientes postinfarto de miocardio (población) la depresión (factor pronóstico) comparando con los pacientes no deprimidos, es un marcador de peor adherencia al tratamiento (resultado)?

\section{Estrategia de búsqueda}

se realizó una búsqueda en Tripdatabase (www.tripdatabase.com) empleando los términos myocardial infarction and depression. Se obtuvieron 3 citas. Se seleccionaron 2 para responder la pregunta.

\section{Resumen de la evidencia}

1)Cita: Frasure-Smith N, Lespérance F, Talajic M. Depression and 18- month prognosis after myocardial infarction. Circulation. 1995 Feb; 999-1005.

Entre agosto de 1991 y julio de 1992 ingresaron a un hospital universitario de Quebec 337 pacientes elegibles para este estudio, de los cuales 227 dieron el consentimiento informado. Cinco pacientes fallecieron antes del alta, por lo tanto la muestra final incluyó 222 pacientes.

Diseño del estudio: cohorte prospectiva que buscaba evaluar la asociación entre depresión y mortalidad cardiovascular luego de un infarto de miocardio. La población se analizó en base a la presencia $o$ ausencia de depresión, definida según 2 parámetros: una entrevista con un investigador para evaluar el diagnóstico de depresión según criterios del DSM-III-R y una entrevista autoadministrada para valorar la presencia o ausencia de síntomas (a través del Beck Depression Inventory). Los pacientes eran entrevistados durante las 2 semanas de ocurrido el infarto. El seguimiento fue a 18 meses.

Medición de resultados: el resultado principal fue la mortalidad cardiovascular y global a 18 meses.

Resultados: La depresión se asoció con la mortalidad global y con la mortalidad cardiovascular a 18 meses (OR 3.64 [IC95\% 1.32 a 10.05], $p=0.012$ y OR 7.82 [IC95\% 2.42 a 25.26], $p=0.001$ respectivamente). En el análisis multivariable*, ajustado por otras covariables, el OR fue de 6.64 (IC 95\% 1.76 a 25.09, p=0.003) para mortalidad cardiovascular.

Conclusión: En pacientes postinfarto, la depresión es un predictor independiente de mortalidad cardiovascular a 18 meses.

2)Cita: Ziegelstein R., Fauerbach J., Stevens S., Romanelli J. Patients With Depression are Less Likely to Follow Recommendations to Reduce Cardiac Risk During Recovery From a Myocardial
Infarction. Arch Intern Med. 2000; 160: 1818-1823.

Durante el período del estudio ingresaron 696 pacientes con diagnóstico de infarto de miocardio, de los cuales 276 eran elegibles. De estos, 204 fueron analizados (seguimiento a 4 meses completo).

Diseño del estudio: es un estudio de cohorte prospectiva que busca determinar si la depresión luego de un infarto de miocardio se asocia con menor adherencia a las recomendaciones para reducir el riesgo de eventos cardiovasculares. Los pacientes fueron entrevistados usando el Beck Depression Inventory (BDI) para valorar la presencia de síntomas de depresión y se usó la Entrevista Clínica Estructurada del DSM-III-R para determinar la presencia de trastorno de la personalidad. La población se analizó según la presencia o ausencia de depresión, definida por éstos 2 parámetros. Los pacientes eran entrevistados entre los 3-5 días de ocurrido el infarto. El seguimiento fue a 4 semanas por medio de una entrevista telefónica usando una escala especial que valora 10 ítems considerados clínicamente relevantes en la adherencia de pacientes postinfarto.

Medición de resultados: los resultados se dividieron en: a) adherencia a 4 meses en pacientes con síntomas de depresión $(\mathrm{BD} \mid>10)$, y $\mathrm{b})$ adherencia a 4 meses en pacientes con depresión mayor y/o distimia (según DSM-III- R). Se analizaron 7 puntos de la escala de adherencia.

Resultados: en el grupo con síntomas de depresión el puntaje de adherencia fue significativamente menor comparado con los pacientes no depresivos $23.0 \pm 5.3$ vs. $26.1 \pm 5.3$; $p<0.01$ ), al igual que en los pacientes con depresión mayor o distimia (20.6 \pm 5.1 vs. $26.1 \pm 5.1 ; p<0.01)$.

\section{Conclusión}

Este estudio muestra que los pacientes con depresión durante la internación por un infarto de miocardio tienen peor adherencia a las recomendaciones realizadas para reducir el riesgo de eventos cardiovasculares.

\section{Comentario}

Ambos estudios son importantes ya que analizan un área pronóstica poco estudiada y muy prevalente (1 de cada 6 pacientes con IAM tienen depresión mayor y al menos 2 de cada 6 tienen síntomas de depresión luego del evento). El primer estudio contesta la pregunta relacionada con la mortalidad y los resultados son contundentes, aunque existen varias explicaciones posibles para esta asociación, a saber:

-anormalidades en el tono autonómico (mayor suceptibilidad para arritmias ventriculares) 
-mayor agregabilidad plaquetaria

-menor tasa de procedimientos de revascularización

- peor adherencia a las recomendaciones para reducir el riesgo de eventos cardiovasculares.

El segundo estudio explora precisamente el último punto, usando una escala de adherencia a las recomendaciones para reducir el riesgo cardiovascular.

Las fortalezas de los estudios incluyen: diseños prospectivos, seguimiento adecuado, cohortes no muy seleccionadas, por lo que sus resultados podrían aplicarse a los pacientes de cualquier Unidad Coronaria de un Hospital de Comunidad.

Los resultados del estudio de Frasure son válidos interna y externamente.

El mecanismo por el cual la depresión se relaciona con la mortalidad no es claro, y podrían jugar un rol varios factores. En este aspecto es en donde el estudio de Ziegelstein cobra importancia, ya que ellos estudian en forma prospectiva si existe asociación entre la depresión y la adherencia al tratamiento.

\section{Resolución del problema}

En base a la evidencia existente podemos concluir que los pacientes con depresión mayor o con síntomas de depresión según el DSM-III-R y el BDI tienen peor pronóstico (mayor mortalidad a 18 meses). La causa de esta mayor mortalidad no es clara. La menor adherencia al tratamiento podría jugar un ro importante aunque la evidencia sobre este punto es menor. De mismo modo que el médico se preocupa por el control de los factores de riesgo vascular clásicos (tabaquismo, hipercolesterolemia, hipertensión, diabetes), debe estar muy atento a la presencia de síntomas de depresión ya que su detección precoz podría modificar el pronóstico del paciente

Dra. Eugenia Natale [ Servicio de Cardiología. Hospital Italiano de Buenos Aires ]
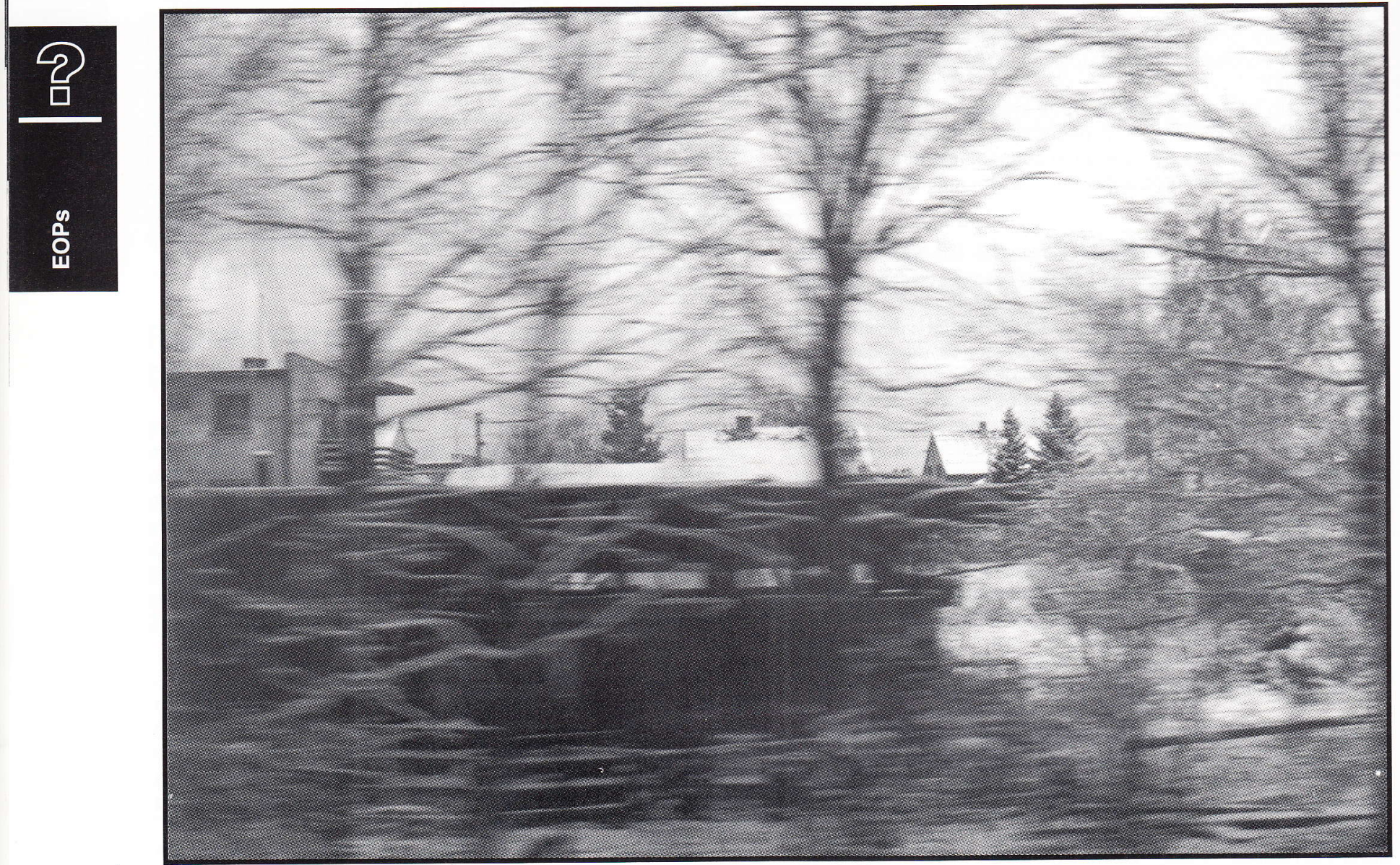